\title{
Recurrent cervical cancer treated with palliative chemotherapy: real-world outcome
}

\author{
Sharada Mailankody1,2a (iD, Manikandan Dhanushkodi ${ }^{1 b}$ (iD, Trivadi S Ganesan ${ }^{1}$, Venkatraman Radhakrishnan ${ }^{1}$, Vasanth Christopher ${ }^{3}$, \\ Selvaluxmy Ganesharajah ${ }^{3}$ and Tenali Gnana Sagar ${ }^{1}$ \\ ${ }^{1}$ Department of Medical Oncology, Cancer Institute (WIA), Adyar, Chennai 600020, India \\ ${ }^{2}$ Department of Medical Oncology, Kasturba Medical College, Manipal Academy of Higher Education, Manipal 576104, India \\ ${ }^{3}$ Department of Radiation Oncology, Cancer Institute (WIA), Adyar, Chennai 600020, India \\ ahttp://orcid.org/0000-0003-2003-426X \\ bhttp://orcid.org/0000-0002-8192-3856
}

\begin{abstract}
Introduction: Cervical cancer is the third most common cancer in India. There is limited data on the treatment of relapsed cervical cancer from India; therefore, we report the outcomes of patients with recurrent cervical cancer who were treated with palliative chemotherapy (CT).
\end{abstract}

Materials and methods: This was a retrospective study of patients with recurrent cervical cancer who received palliative CT from January 2012 to December 2016. The demographic details, clinical profile and survival outcomes were collected. Patients were treated with carboplatin or paclitaxel and carboplatin. Local radiation was given for symptomatic patients. Patients were assessed for responses clinically and/or radiologically after three and six cycles of CT. Progression-free survival (PFS) and overall survival (OS) were calculated using the Kaplan-Meier method.

Results: Forty-six patients with recurrent cervical cancer were included in this analysis, with a median follow-up of 9.4 months. The median age was 49.5 (25-65) years and the median disease-free interval was 31.3 (2-196) months. Biopsy confirmation of relapse was established in $63 \%$. The median number of CT cycles was six. Twenty-four (52.2\%) patients completed six cycles of CT. The overall response rate was $56.5 \%$. Patients with a complete or a partial response were more likely to have PFS $>6$ months $(p<0.0001)$. Median PFS and OS were, respectively, $8.4(95 \% \mathrm{Cl} 6.1-10.7)$ months and $10.3(95 \% \mathrm{Cl}$ 6.8-13.8) months. The completion of all cycles of CT and the site of metastasis (nodal vs. visceral or combined) were found to be associated with OS.

Conclusion: Palliative CT with paclitaxel carboplatin is a safe and effective option in Indian patients with recurrent cervical cancer, with more than half of the patients completing the prescribed CT. Further prospective trials may be required to place this treatment in the right context, in this era of immunotherapy and targeted therapy. However, knowing the outcomes in our population and prognostic factors will help in better prognostication of patients, thereby channelling our limited resources where necessary.

Keywords: chemotherapy, recurrent cervical cancer, palliative, outcomes, prognostic factors
Correspondence to: Manikandan Dhanushkodi Email: dmani1982@gmail.com

ecancer 2020, 14:1122

https://doi.org/10.3332/ecancer.2020.1122

Published: $13 / 10 / 2020$

Received: 07/05/2020

Publication costs for this article were supported by ecancer (UK Charity number 1176307).

Copyright: (c) the authors; licensee ecancermedicalscience. This is an Open Access article distributed under the terms of the Creative Commons Attribution License (http:// creativecommons.org/licenses/by/3.0), which permits unrestricted use, distribution, and reproduction in any medium, provided the original work is properly cited. 


\section{Introduction}

Cervical cancer continues to be a significant cause of morbidity and mortality in India, despite the introduction of screening strategies in the community [1, 2]. It is the third most common cancer in India. There are 10,000 new cases registered per year, and it also has a higher mortality rate in India [3, 4]. It is second only to breast cancer among the causes of cancer mortality among females in 12 of the Indian states [1].

In India, the epidemiology of the disease has a distinct pattern, with more advanced stages at presentation and a definite rural predominance $[1,5,6]$. Also, the availability and organisation of oncological care are different in lower and middle-income countries (LMICs) compared to high-income countries, especially when it comes to medical oncology services [7]. Hence, the management of recurrent cervical cancer poses unique challenges in India and merits exploration.

The treatment of recurrent cervical cancer may require multimodality care, with chemotherapy (CT)/immunotherapy, radiotherapy (RT) or surgery; the exact sequence of which has not been established as yet $[8,9]$. The prognosis of recurrent cervical cancer depends on multiple factors like performance status (PS), prior cisplatin use, pelvic disease, race, sites of metastasis and disease-free interval (DFI) [10-13]. With the approval of immunotherapy for recurrent cervical cancer, systemic treatment options now include platinum-based doublet CT, bevacizumab and immunotherapy [8]. Also, in resource-limited settings, the use of oral metronomic chemotherapy (OMC) has been described [14]. The overall response rates (ORR) from 15\% to 65\% have been described for various protocols in various settings of care [15-24]. There is paucity of data regarding the outcomes of systemic treatment of recurrent cervical cancer from India [20]. It is important to know the prognostic factors and outcomes of treatment so that our limited resources can be channelled towards treating the right patients. We undertook this analysis to explore the outcomes of CT in patients presenting with recurrent cervical cancer.

\section{Materials and methods}

We collected data from the individual case records of patients with recurrent cervical cancer who were treated with palliative CT from January 2012 to December 2016. The demographic details, clinical profile and the treatment details were collected. The recurrence was confirmed with a fine needle aspiration or biopsy for those with late recurrences in accessible sites. The restaging investigation was by either contrast-enhanced computed tomography or positron emission tomography/computed tomography .

\section{Treatment protocol}

After a multidisciplinary tumour board (MDT) discussion, patients were treated with palliative CT, with either single-agent carboplatin or paclitaxel and carboplatin (PC). The dose was carboplatin (area under the curve (AUC) 5) or paclitaxel (175 mg/m²) and carboplatin (AUC 5) every 3 weeks for six cycles depending on age, comorbid illness and PS. Patients were assessed for responses clinically and radiologically after three and six cycles of CT. Palliative radiation was given when indicated.

\section{Statistical analysis}

Progression-free survival (PFS) was calculated from the date of starting CT until the date of last follow-up, progression or death. Overall survival (OS) was calculated from the date of starting CT until the date of last follow-up or death due to any cause. Follow-up was censored in August 2017. Actuarial survival was computed using the Kaplan-Meier method; prognostic factors were compared using the log-rank test. Multivariate analysis was carried out using the Cox proportional hazards regression model. All statistical analyses were carried out using SPSS version 13 (IBM Inc.), and a $p$-value $<0.05$ was considered to be significant. 


\section{Results}

\section{Baseline characteristics}

A total of 46 patients with recurrent cervical cancer were included in this analysis (Table 1).

Median age at presentation was 49.5 years (range: $25-65$ years). The stages at presentation were stage I: 5 patients (10.9\%); stage II: 32 patients (69.6\%) and stage III: 9 patients (19.6\%). All patients were treated with pelvic external beam radiotherapy with $45-50$ Gy, followed by three high-dose-rate intracavitary applications done to deliver 21 or 24 Gy at point A. Target Eqd2 aimed was 80 Gy. Concurrent CT with weekly cisplatin was given to 37 patients (80.4\%). Parametrial radiotherapy was given to 29 (63\%) patients and paraaortic RT to 7 (15.2\%) patients.

Median DFI was 31.3 months (range: $2-196$ months). The DFI was less than a year in $19.6 \%(n=9)$ and more than a year in $80.4 \%(n=37)$. Asymptomatic and symptomatic relapses were $67.4 \%(n=31)$ and $32.6 \%(n=15)$, respectively. Single site of relapse was seen in $16(34.8 \%)$ patients. The sites of relapse were lung in 27 (58.7\%) patients, distant nodes in 24 (52.2\%) patients, paraaortic nodes in 21 (45.7\%) patients, local in 5 (10.9\%) patients, bone in $5(8.7 \%)$ patients and liver/adrenal in $3(6.5 \%)$ patients. Of the patients with node-only metastasis $(n=$ $13,28.3 \%)$, five (10.9\%) patients had paraaortic and supraclavicular metastasis, five (10.9\%) patients had isolated paraaortic and one (2.2\%) patient each had supraclavicular node, mediastinal node and inguinal node relapse. Tissue confirmation of recurrence was available in 29 (63\%) patients.

The CT delivered was PC to 40 (87\%) patients and single-agent carboplatin to 6 (13\%) patients. Four (8.7\%) patients were started initially on single-agent carboplatin, and then escalated to PC from the second cycle. Bevacizumab was given to one patient. The median number of treatment cycles was 6 (range: 1-6 cycles) and 24 (52.2\%) patients completed all six cycles. Palliative radiation was given to 20 (43.5\%) patients; except two patients with isolated local recurrence who were given pelvic/local RT, the other patients $(n=18)$ were given RT to the site of relapse.

Table 1. Baseline characteristics of patients with recurrent cervical cancer.

\begin{tabular}{|l|c|}
\hline \multicolumn{1}{|c|}{ Characteristic } & No of patients (\%) (n= 46) \\
\hline Age (median(range)) & $49.5(25-65)$ \\
\hline Stage* & $5(10.9)$ \\
\hline I & $32(69.6)$ \\
\hline II & $9(19.6)$ \\
\hline III & \multicolumn{2}{|c|}{} \\
\hline Grade ${ }^{*}$ & $5(10.9)$ \\
\hline II & $41(89.1)$ \\
\hline III & \\
\hline Histology* & $37(80.4)$ \\
\hline SCC & $4(8.7)$ \\
\hline PDC & $5(10.9)$ \\
\hline Adenocarcinoma & $37(80.4)$ \\
\hline Cisplatin* & $29(63)$ \\
\hline Parametrial RT* & $7(15.2)$ \\
\hline Paraaortic RT* & 2.6 \\
\hline Mean ICAs* & \\
\hline
\end{tabular}

*Data presented as $n(\%)$ 


\section{Response rates and survival outcomes}

The ORR was 56.5\%, with a complete response in 5 (10.9\%) patients (Figure 1). Patients with response (CR/PR) to CT were more likely to have a survival of $>6$ months ( $p<0.00001$ ). At the end of the study period, 15 (32.6\%) patients were alive. The median follow-up was 9.4 months (range: 1.1-52.6). The median PFS and OS rates were 8.4 (95\% Cl 6.1-10.7) months and 10.3 (95\% Cl 6.8-13.8) months, respectively (Figure 2). The rates of one-year PFS and OS were $34.3 \%$ and $48.4 \%$, respectively.

\section{Factors affecting OS}

On univariate analysis, the response to CT, site of metastasis and the completion of CT correlated with OS (Table 2). The patients with nodeonly metastasis were found to have a better OS than patients with visceral metastases or combined visceral-nodal metastasis. On multivariate analysis, the factors affecting survival were completion of CT and metastasis sites (Figure 3). Age, stage, tumour histology, grade, DFI and addition of RT did not correlate with survival.

\section{Toxicity details}

Twenty-one (45.7\%) patients had CT-related side effects, with 14 (30.4\%) patients having neuropathy. Grade 3/4 side effects were seen in six (13\%) patients, two (4.3\%) patients with neuropathy, two (4.3\%) patients with myelosuppression and two (4.3\%) patients with metabolic complications. There was one death due to sepsis; however, this was in a non-neutropenic setting.

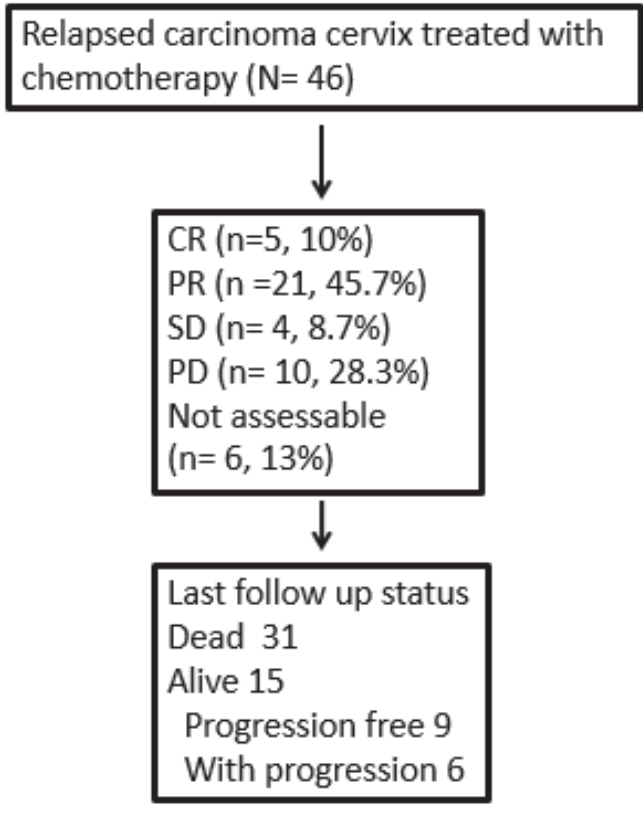

Figure 1. Responses and outcomes of patients with relapsed cervical cancer. 

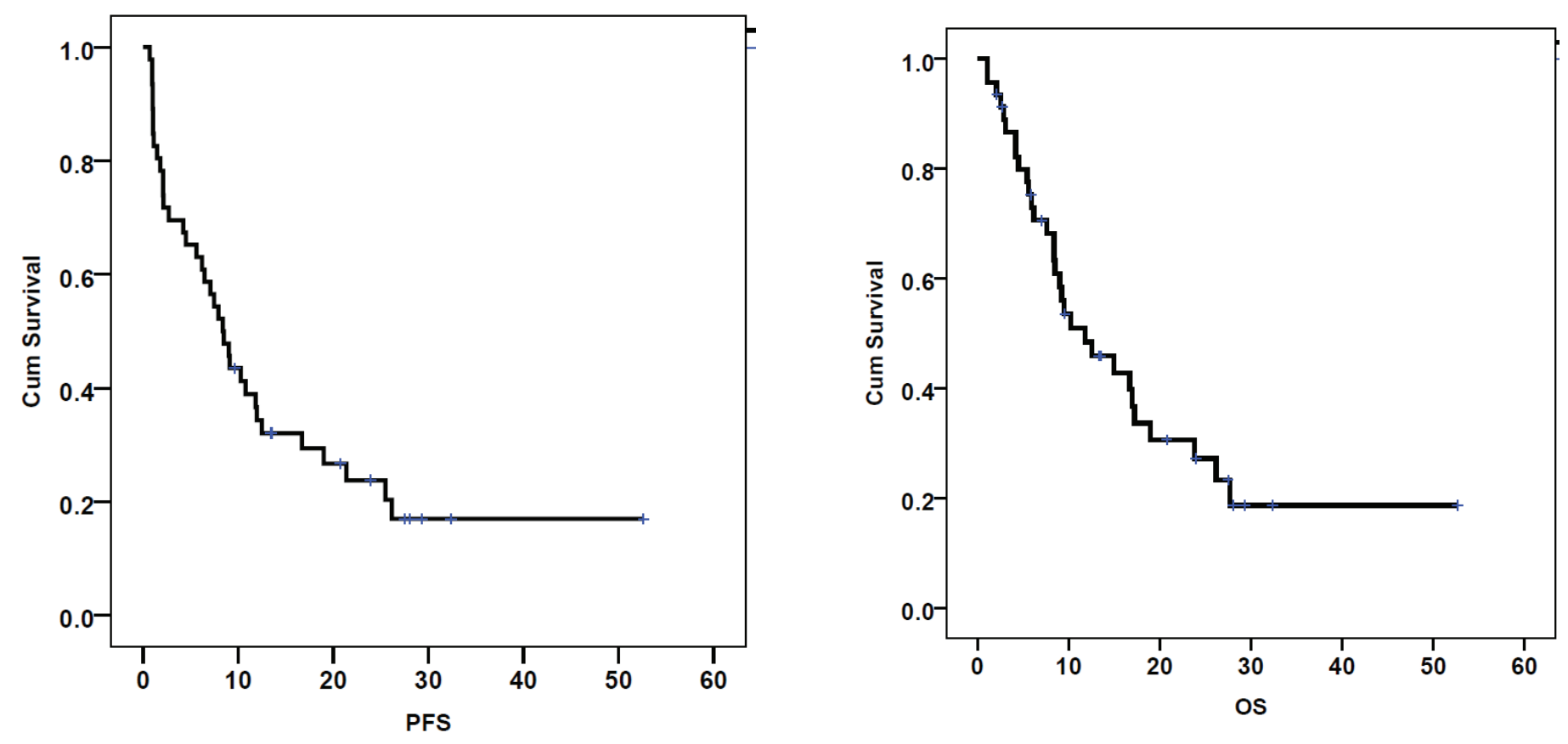

Figure 2. PFS and OS calculated using the Kaplan-Meier curves in patients with recurrent cervical cancer treated with palliative chemotherapy ( $n=46$ ).

\section{Discussion}

This study reports the outcomes of CT in recurrent cervical cancer from a tertiary cancer care hospital in India. With CT, there was an ORR of $56.5 \%$, with CR in $10.9 \%$ patients. The median PFS and OS were 8.4 months and 10.3 months, respectively. Half of the patients (52.2\%) were able to complete six cycles of $\mathrm{CT}$. We also identified prognostic factors associated with survival.

A study in Japan found an RR of 70\% in a trial using PC for recurrent cervical cancer, with a median PFS of 7 months [16]. In another Japanese trial, comparing PC with paclitaxel/cisplatin, an RR of $62.6 \%$ with median PFS and OS of 6.2 months and 17.5 months, respectively, was reported [17]. However, while the current study had $80 \%$ of the patients exposed to cisplatin, the above-mentioned trial included only $48 \%$ of such patients. In a systematic review comparing cisplatin and carboplatin-based therapies, an RR of $48.5 \%$ and mean PFS and OS of 5 months and 10 months, respectively, was seen for carboplatin-based therapy [18]. In another Indian study analysing the outcomes of both pre-treated and de-novo metastatic cervical cancer with PC, an RR of 26.7\% with a median PFS of 6 months and OS of 11 months was reported [20].

The RR in our study was higher than that reported by the GOG trial which used cisplatin-based doublets and other retrospective studies $[20,21,25]$. However, our analysis did not include patients with persistent disease after the completion of chemoradiation therapy. Moreover, only around $20 \%$ of the patients in our study had a DFI of $<6$ months.

Although nearly half of the patients developed toxicities, there was only one toxic death. While neuropathy was the most significant toxicity in our study (30\% of the patients), only two patients had grade 3 or 4 neuropathy. Although a Brazilian study has also reported $30 \%$ neuropathy with the use of PC, this percentage is higher than that reported by few other studies [17, 24]. Prior cisplatin treatment in 80\% of our patients may account for the higher rates of neuropathy in our patients. 
Interestingly, with the use of single-agent OMC (etoposide or cyclophosphamide) in a similar population, an RR of $23 \%$ and a median OS of 14.5 months was reported [14]. Since our study used two active agents, the RR may have been higher. The survival time seems similar across these studies.

In the current study, patients with node-only metastasis had a significantly better survival compared to those with visceral metastasis. Also, there was an improved survival in those who completed chemotherapy. Not surprisingly, the patients with CR/PR also had longer survival. These factors were reported in prior studies also [11-13]. However, age, histology, grade, DFI and prior cisplatin use were not found to be prognostic in the current study, which is contrary to the earlier studies [10].

Table 2. Results of univariate and multivariate analysis of factors affecting survival.

\begin{tabular}{|c|c|c|c|}
\hline Factor & OS (95\% Cl) & $\begin{array}{c}p \text { value } \\
\text { (for univariate analysis) }\end{array}$ & $\begin{array}{c}p \text { value } \\
\text { (for multivariate analysis) }\end{array}$ \\
\hline \multicolumn{4}{|l|}{ Completion of CT } \\
\hline Yes $(n=24)$ & $23.8(13.6-34.1)$ & \multirow{2}{*}{0.000} & \multirow{2}{*}{0.000} \\
\hline No $(n=22)$ & $5.9(1.7-10.1)$ & & \\
\hline \multicolumn{4}{|l|}{ Site of metastasis* } \\
\hline Nodal $(n=13)$ & $26.2(11.8-40.5)$ & \multirow{3}{*}{0.034} & \multirow{3}{*}{0.001} \\
\hline Visceral $(n=9)$ & $9.2(7.3-11.1)$ & & \\
\hline Combined $(n=22)$ & $8.4(2.0-14.8)$ & & \\
\hline \multicolumn{4}{|l|}{ Response to therapy** } \\
\hline SD/PD $(n=15)$ & $5.9(2.4-9.4)$ & \multirow{2}{*}{0.028} & \multirow{2}{*}{0.843} \\
\hline CR/PR $(n=26)$ & $17.2(11.8-22.6)$ & & \\
\hline \multicolumn{4}{|l|}{ DFI } \\
\hline Less than 1 year $(n=9)$ & $8.4(0.0-18.6)$ & \multirow{2}{*}{0.106} & \multirow{2}{*}{ NA } \\
\hline More than 1 year $(n=37)$ & $14.9(6.7-23.2)$ & & \\
\hline \multicolumn{4}{|l|}{ Age } \\
\hline Less than 50 years $(n=21)$ & $14.9(6.4-23.5)$ & \multirow{2}{*}{0.305} & \multirow{2}{*}{ NA } \\
\hline More than 50 years $(n=25)$ & $9.0(3.8-14.2)$ & & \\
\hline \multicolumn{4}{|l|}{ Symptomatic relapse } \\
\hline Yes $(n=15)$ & $9.2(6.1-12.4)$ & \multirow{2}{*}{0.191} & \multirow{2}{*}{ NA } \\
\hline No $(n=31)$ & $12.6(2.6-22.6)$ & & \\
\hline \multicolumn{4}{|l|}{ Histology } \\
\hline Squamous ( $n=37)$ & $11.8(5-18.6)$ & \multirow{2}{*}{0.598} & \multirow{2}{*}{ NA } \\
\hline Non $\operatorname{SCC}(n=9)$ & $17.2(7.6-26.8)$ & & \\
\hline \multicolumn{4}{|l|}{ Cisplatin upfront } \\
\hline Yes $(n=37)$ & $9.5(4.8-14.2)$ & \multirow{2}{*}{0.628} & \multirow{2}{*}{ NA } \\
\hline No $(n=9)$ & $14.9(5.7-24.2)$ & & \\
\hline
\end{tabular}

*Excluding the two patients with isolated local recurrence

**Excluding the five patients whose response could not be assessed 

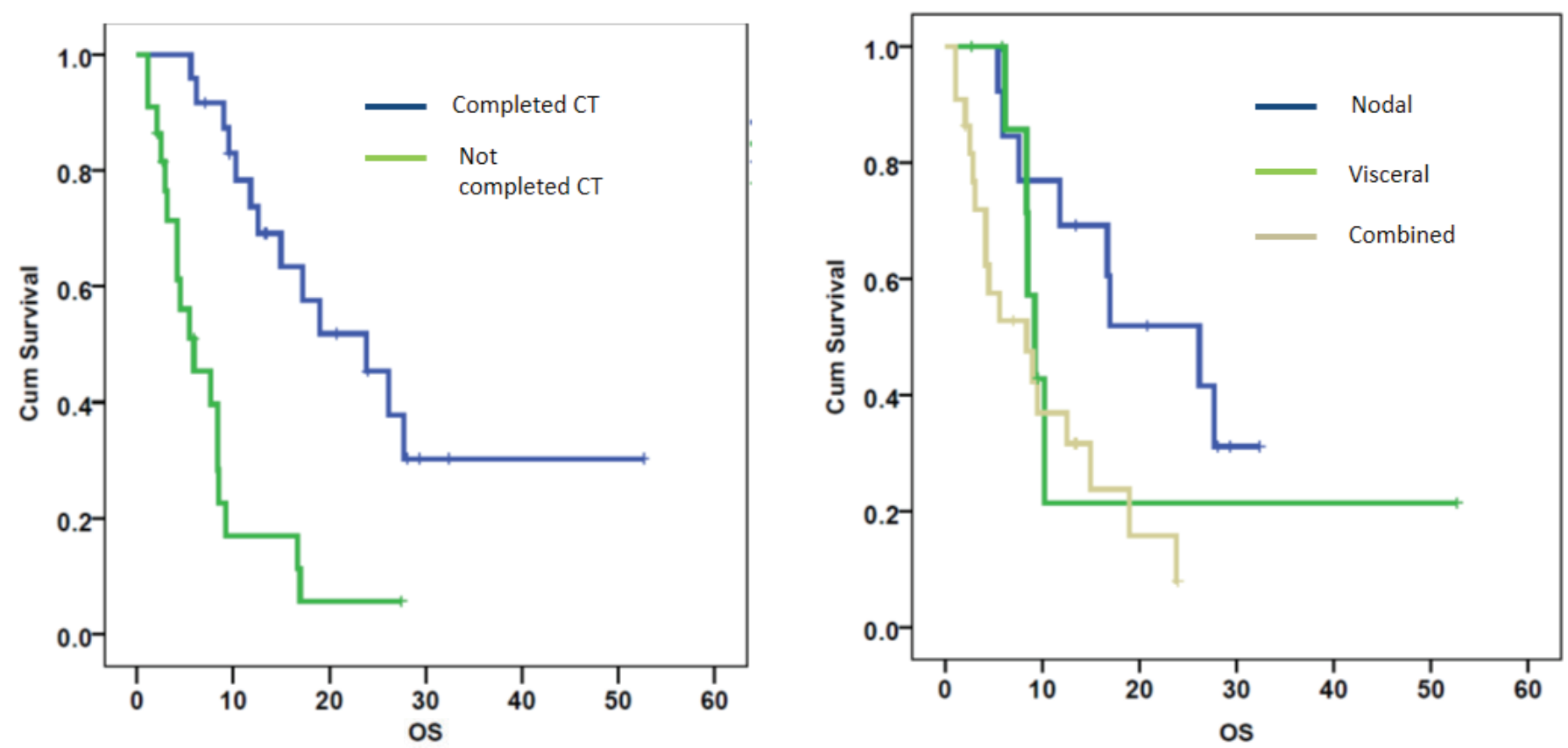

Figure 3. OS calculated using the Kaplan-Meier curves for patients based on a) completion of all cycles of chemotherapy (CT) ( $p=0.000)$ and b) site of metastasis $(p=0.001)$.

Table 3. Toxicity details in patients given palliative chemotherapy.

\begin{tabular}{|l|c|}
\hline \multicolumn{1}{|c|}{ Toxicity } & No of patients (\%) \\
\hline Neuropathy & $12(26)$ \\
\hline Grade 1/2 & $2(4)$ \\
\hline Grade 3/4 & \\
\hline Haematological & $2(4)$ \\
\hline Grade 3/4 myelosupression & $1(2)$ \\
\hline Grade 2 thrombosis & $1(2)$ \\
\hline Grade 3 colonic perforation & $1(2)$ \\
\hline Grade 2 renal dysfunction & $1(2)$ \\
\hline Grade 3 dyselectrolytemia & $1(2)$ \\
\hline Death & \\
\hline
\end{tabular}

This study represents the real-world outcome of recurrent cervical cancer treated with palliative CT. Cervical cancer in India is rurally predominant; hence, expensive treatment options, like immunotherapy and bevacizumab, may not be cost-effective, although low-cost biosimilar bevacizumab molecules are now available. Many patients have to travel long distances to reach oncology centres, thus developing and optimising simple yet effective protocols are very important for treatment compliance [7]. Moreover, the out-of-pocket expenditures are high for the treatment of cancer in India; although the cost of CT may be subsidised or free, the treatment process may cripple the family financially [25]. Hence, knowing the outcomes in our country and prognostic factors may help us to choose the appropriate patients for the initiation of palliative CT. 
Further data are needed regarding the outcomes and ideal protocols of OMC with pharmacokinetics in this population, as we have in head and neck cancer patients [26]. However, till such data become available, the use of well-tolerated simple CT protocols may form the cornerstone for effective palliation of such patients.

The limitations of this study are retrospective design, small sample size and limited use of targeted therapy.

\section{Conclusion}

PC is a useful treatment option in patients with recurrent cervical cancer. Lymph nodal metastasis and completion of therapy were associated with better outcomes. Further prospective studies are needed in India and other LMICs to exactly define the role of CT and the selection of the right patients for initiation of treatment. Identification of outcomes and prognostic factors in our population will help us in the optimal utilisation of available resources.

\section{List of abbreviations}

LMICs Lower- and middle-income countries

DFI Disease-free interval

OMC Oral metronomic chemotherapy

AUC Area under the curve

PS Performance status

CR Complete response

PR Partial response

PFS Progression-free survival

OS Overall survival

ORR Overall response rate

PC Paclitaxel carboplatin

CT Chemotherapy

\section{Conflicts of interest}

None of the authors have any conflict of interest.

\section{Funding}

None.

\section{References}

1. India State-Level Disease Burden Initiative Cancer Collaborators The burden of cancers and their variations across the states of India: the Global Burden of Disease Study 1990-2016 Lancet Oncol 19(10) 1289-1306

2. Srivastava AN, Misra JS, and Srivastava S, et al (2018) Cervical cancer screening in rural India: status \& current concepts Indian J Med Res 148(6) 687-696 https://doi.org/10.4103/ijmr.IJMR_5_17 
3. GLOBOCAN 2018 [https://gco.iarc.fr/today/data/factsheets/populations/356-india-fact-sheets.pdf] Date accessed: 20/04/20

4. Torre LA, Islami F, and Siegel RL, et al (2017) Global cancer in women: burden and trends Cancer Epidemiol Biomarkers Prev 26(4) 444-457 https://doi.org/10.1158/1055-9965.EPI-16-0858 PMID: 28223433

5. Bobdey S, Sathwara J, and Jain A, et al (2016) Burden of cervical cancer and role of screening in India Indian J Med Paediatr Oncol 37(4) 278-285 https://doi.org/10.4103/0971-5851.195751

6. Thakur JS, Budukh A, and Kapoor R, et al (2017) Urban-rural differences in cancer incidence and pattern in Punjab and Chandigarh: findings from four new population-based cancer registries in North India Int J Non-Commun Dis 2 49-55

7. Sengar M, Fundytus A, and Hopman WM, et al (2019) Medical oncology in India: workload, infrastructure, and delivery of care Indian J Med PaediatrOncol 40 121-127 https://doi.org/10.4103/ijmpo.ijmpo_66_18

8. Li H, Wu X, and Cheng X (2016) Advances in diagnosis and treatment of metastatic cervical cancer J GynecolOncol 27(4) e43

9. Kranawetter M, Röhrich S, and Müllauer L, et al (2018) Activity of pembrolizumab in recurrent cervical cancer: case series and review of published data Int J Gynecol Cancer 28(6) 1196-1202 https://doi.org/10.1097/IGC.0000000000001291 PMID: 29787422

10. Tewari KS, Sill MW, and Monk BJ, et al (2015) Prospective validation of pooled prognostic factors in women with advanced cervical cancer treated with chemotherapy with/without bevacizumab: NRG Oncology/GOG Study Clin Cancer Res 21(24) 5480-5487 https:// doi.org/10.1158/1078-0432.CCR-15-1346 PMID: 26672085 PMCID: 4896296

11. Kim K, Cho SY, and Kim BJ, et al (2010) The type of metastasis is a prognostic factor in disseminated cervical cancer J Gynecol Oncol 21(3) 186-190 https://doi.org/10.3802/jgo.2010.21.3.186 PMID: 20922142 PMCID: 2948227

12. Yin Z, Tang H, and Li L, et al (2019) Impact of sites versus number of metastases on survival of patients with organ metastasis from newly diagnosed cervical cancer Cancer Manag Res 11 7759-7766 https://doi.org/10.2147/CMAR.S203037 PMID: 31496818 PMCID: 6701644

13. Sasano T, Mabuchi S, and Kuroda $\mathrm{H}$, et al (2016) Predictors of survival in patients with FIGO stage IVB cervical cancer Int J Gynecol Cancer 26(3) 528-533 https://doi.org/10.1097/IGC.0000000000000642 PMID: 26825839

14. Baruah U, Barmon D, and Hazarika M, et al (2014) Continuous low-dose oral chemotherapy in recurrent and persistent carcinoma of cervix following chemoradiation: a comparative study between prolonged oral cyclophosphamide and oral Etoposide Indian J Palliat Care 20(3) 208-211 https://doi.org/10.4103/0973-1075.138396 PMID: 25191008 PMCID: 4154168

15. Kumar L and Gupta S (2016) Integrating chemotherapy in the management of cervical cancer: a critical appraisal Oncology 91(suppl 1) 8-17 https://doi.org/10.1159/000447576 PMID: 27464068

16. Mabuchi S, Morishige K, and Fujita M, et al (2009) The activity of carboplatin and paclitaxel for recurrent cervical cancer after definitive radiotherapy Gynecol Oncol 113(2) 200-204 https://doi.org/10.1016/j.ygyno.2009.02.008 PMID: 19268342

17. Kitagawa R, Katsumata N, and Shibata T, et al (2015) Paclitaxel plus carboplatin versus paclitaxel plus cisplatin in metastatic or recurrent cervical cancer: the open-label randomized phase III trial JCOG0505 J Clin Oncol 33(19) 2129-2135 https://doi.org/10.1200/ JCO.2014.58.4391 PMID: $\underline{25732161}$

18. Lorusso D, Petrelli F, and Coinu A, et al (2014) A systematic review comparing cisplatin and carboplatin plus paclitaxel-based chemotherapy for recurrent or metastatic cervical cancer Gynecol Oncol 133(1) 117-123 https://doi.org/10.1016/j.ygyno.2014.01.042 PMID: 24486604

19. Bonanthaya R, Lakshmaiah K, and Babu S, et al (2016) Palliative chemotherapy in recurrent carcinoma cervix: experience from a regional cancer centre in southern India Southern Afr J Gynaecol Oncol 8(1) 14-17 https://doi.org/10.1080/20742835.2016.1175152

20. Lakshmaiah KC, Thanky AH, and Lokanatha D, et al (2017) Epidemiology and outcomes with platinum-based chemotherapy in recurrent or metastatic carcinoma cervix in a developing country: experience from a Tertiary Oncology Centre in Southern India. Gulf $J$ Oncolog 1(25) 20-26 PMID: 29019326 
21. Monk BJ, Sill MW, and McMeekin DS, et al (2009) Phase III trial of four cisplatin-containing doublet combinations in stage IVB, recurrent, or persistent cervical carcinoma: a Gynecologic Oncology Group study J Clin Oncol 27(28) 4649-4655 https://doi.org/10.1200/ JCO.2009.21.8909 PMID: 19720909 PMCID: 2754911

22. Choi HJ, Paik ES, and Choi $\mathrm{CH}$, et al (2018) Response to combination chemotherapy with paclitaxel/ifosfamide/platinum versus paclitaxel/platinum for patients with metastatic, recurrent, or persistent carcinoma of the uterine cervix: a retrospective analysis Int J Gynecol Cancer 28(7) 1333-1341 https://doi.org/10.1097/IGC.0000000000001316 PMID: 30045137

23. Ruengkhachorn I, Leelaphatanadit C, and Therasakvichya S, et al (2016) Oncologic outcomes of stage IVB or persistent or recurrent cervical carcinoma patients treated with chemotherapy at Siriraj Hospital: Thailand's largest tertiary referral center Int J Gynecol Cancer 26(6) 1154-1161 https://doi.org/10.1097/IGC.0000000000000712 PMID: 27051060 PMCID: 4920274

24. Garces ÁH, Mora PA, and Alves FV, et al (2013) First-line paclitaxel and carboplatin in persistent/recurrent or advanced cervical cancer: a retrospective analysis of patients treated at Brazilian National Cancer Institute Int J Gynecol Cancer 23(4) 743-748 https://doi. org/10.1097/IGC.0b013e31828c141d PMID: 23552805

25. Rajpal S, Kumar A, and Joe W. (2018) Economic burden of cancer in India: evidence from cross-sectional nationally representative household survey 2014 PLoS One 13(2) e0193320 https://doi.org/10.1371/journal.pone.0193320 PMCID: 5826535

26. Patil VM, Noronha V, and Joshi A, et al (2019) Phase I/II study of palliative triple metronomic chemotherapy in platinum-refractory/ early-failure oral cancer J Clin Oncol 37(32) 3032-3041 https://doi.org/10.1200/JCO.19.01076 PMID: 31539316 